Physics

Electricity \& Magnetism fields

Okayama University

Year 1991

\title{
Factors affecting errors due to 2-D approximate analysis of 3-D magnetic fields with eddy currents [rotating machines]
}

\author{
Norio Takahashi \\ Okayama University \\ K. Fujiwara \\ Okayama University
}

\author{
Takayoshi Nakata \\ Okayama University \\ T. Nishimura \\ Okayama University
}

This paper is posted at eScholarship@OUDIR : Okayama University Digital Information Repository.

http://escholarship.lib.okayama-u.ac.jp/electricity_and_magnetism/81 
FACTORS AFFECTING ERRORS DUE TO 2-D APPROXIMATE ANALYSIS

OF 3-D MAGNETIC FIELDS WITH EDDY CURRENTS

N.Takahashi, T.Nakata, K.Fujiwara and T.Nishimura

Department of Electrical Engineering, Okayama University

Okayama 700, Japan

Abstract - The influences of the fringing from the side of core, the eddy current path and the end coil of rotating achines on the flux and eddy current distributions analyzed by the 2-D numerical method are investigated systenatically by comparing 2-D analysis with 3-D analysis. It is shown that the error in 2-D analysis is significant when the gap length in the magnetic circuit is large and the eddy current flows in the core. The numerical results are also verified experimentally.

\section{INTRODUCTION}

When rotating machines with eddy currents, such as induction motors, are analyzed approximately as 2-D models, some error occurs when the axial length of the machine is relatively short. This may be due to the following reasons:

a) The leakage flux from the side of the core is not correctly wodeled by 2-D analysis.

b) There is a great difference in eddy current paths between 2-D and 3-D models [1].

c) The magnetomotive force produced by the end coil is not considered in $2-D$ analysis.

It is inportant to examine the minimum length (thickness) of the machine which can be approximated by 2-D analysis. A systematic analysis, however, has not been undertaken until now.

In this paper, the effects of the fringing from the side of the core, the eddy current path and the end coil on the flux and eddy current distributions are investigated systematically by comparing 2-D and 3-D analyses. Experimental investigation is also carried out using various shapes of models in order to verify the numerical analysis.

\section{MODELS AND METHOD OF ANALYSIS}

A. Description of models

Fig. 1 shows a 3-D basic model for rotating machines to be investigated. Two pole pieces of semiinfinite length in the $z$-direction are put inside a coil of infinite length. The coil is excited from a constant current or voltage source $[2,3]$. In order to examine only factors mentioned in section $I$, the agnetic characteristic of the solid pole pieces is

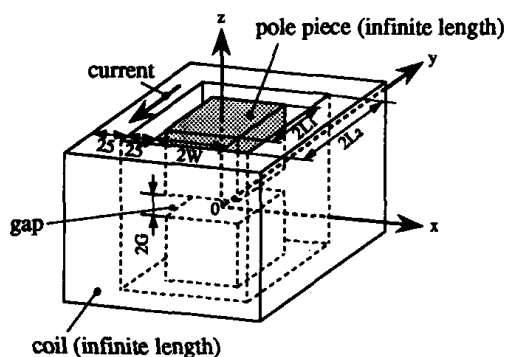

Fig.1 Analyzed model. assumed to be linear and the relative permeability is taken to be 1000 .

The basic model is modified to the models shown in Figs. 2 and 3 in order to investigate the effect of only one of the following factors which affects the flux and eddy current distributions:

1) Fringing from side of pole piece

The effect of the leakage flux from the side of the pole piece is investigated by comparing the two models shown in Fig.2. Model $A$ is a 2-D model $(x-z)$ which has no side effect in the $y$-direction. It means that the widths $L_{1}$ and $L_{2}$ of the pole piece and the coil in Fig. 1 are both infinite. As the shape of the pole piece of the model $B$ is three-dimensional, the flux leaks out from the side of the pole piece. In order to avoid the effects of the eddy current and the coil end, the coil is excited from a dc source and the width L2 of the coil is chosen as infinite in the $y$-direction. The fringing effect is investigated by changing the gap length $\mathrm{G}$.

The Neumann boundary condition is imposed on the line $0-a-b-c$, and the Dirichlet boundary condition is put on the line $0-c$ in the model $A(2-D)$ in Fig.2(a). The boundary condition that the flux flows parallel to the boundary[4] is given on the planes $0-a-b-c-0,0-d-g-c-0$ and $d-e-f-g-d$ in the model $B(3-D)$ in Fig.2(b). The boundary condition that the flux is perpendicular to the boundary[4] is given on other planes.

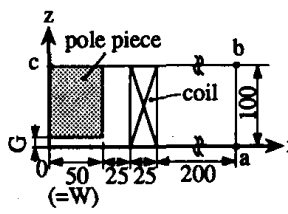

(a) model A (2-D)

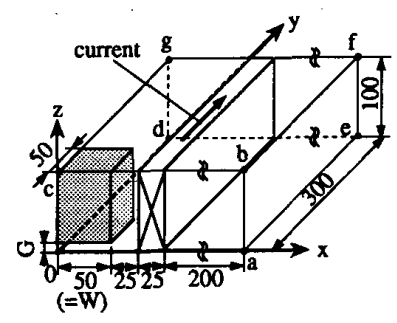

(b) model B (3-D)
Fig. 2 Models for investigating fringing effect.

2) Eddy current path

The effect of eddy current path is investigated by comparing the two models shown in Fig.3. In order to avoid the fringing effect, the gap length $G$ is chosen as zero. The fringing flux from the side becomes zero when there is no gap. The width $\mathrm{L}_{2}$ of the

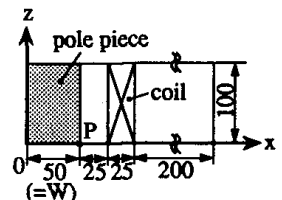

(a) model C (2-D)

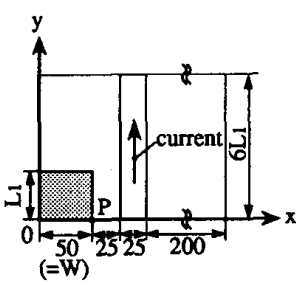

(b) model D (3-D)
Fig.3 Models for investigating effect of eddy current path. 
coil in Fig. 1 is chosen as infinite so that the effect of the magnetomotive force produced by the $x$-component of the current is removed. The model $C$ is twodimensional $(x-z)$. The model $D$ is also twodimensional $(x-y)$, but it can be used to investigate the effect of the 3-D eddy current path on the flux and eddy current distributions by changing the width $\mathrm{L}_{1}$ of the core. The frequency of the ac source is $60 \mathrm{~Hz}$. The eddy current in the coil is neglected.

The same boundary conditions as the models in Fig.2 are inposed in Fig.3.

B. Method of analysis

The A method, in which the magnetic vector potential $A$ is chosen as an unknown variable, and the first-order edge brick element[5] are used in the finite element analysis.

In the analysis of the eddy current models $C$ and $D$, the region over which the distance from the surface is equal to the skin depth $\delta$ is subdivided into six layers of elements in order to analyze accurately the eddy current distribution.

\section{III . FACTORS AFFECTING ERROR}

A. Fringing from side of pole piece

Fig.4 shows the effect of the gap length $G$ on the fringing flux from the side of the pole piece. $\phi(2)$ and $\phi(3)$ are the fluxes which pass through the pole pieces in the 2-D model $A$ and the 3-D model $B$ respectively. These are the fluxes per $50 \mathrm{~mm}(=W)$ in the $y$-direction at $z=G+2 m$.

Under the constant voltage excitation[2], $\phi(3)$ is less than $\phi(2)$. This is due to the difference of the fringing effect. The total flux in the region c'$b^{\prime}-f^{\prime}-g^{\prime}-c^{\prime}$ in Fig.5(a) is equal to that in the region $\mathrm{c}-\mathrm{b}-\mathrm{f}-\mathrm{g}-\mathrm{c}$ in Fig.5(b). As the fringing flux exists around the pole piece in the 3-D mode1, the flux $\phi(3)$ is less than $\phi(2)$ as shown in Fig. 5 .

Under the constant current excitation, $\phi(3)$ in Fig.5 is larger than $\phi(2)$ contrary to the case under the constant voltage excitation. This is due to the decrease of the magnetic resistance for the $3-\mathrm{D}$ model.

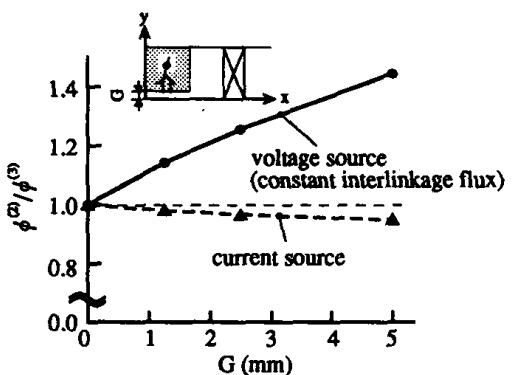

Fig.4 Effect of gap length $G$ on flux in pole piece (dc).

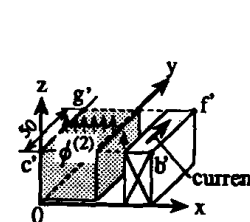

(a) model A (2-D)

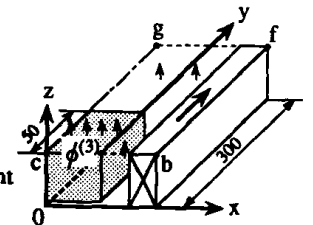

(b) model B (3-D)
Fig.5 Flux distributions under constant voltage excitation.
B. Eddy current path

Fig. 6 shows the effects of the width of the core, $L_{1}$, on the ratios of the flux $\phi(2) / \phi(3)$ and the eddy current density $\mathrm{Je}_{\mathrm{e}}(2) / \mathrm{Je}_{\mathrm{e}}(3)$ under the constant current excitation. $\mathrm{Je}_{e}(2)$ and $\mathrm{Je}^{(3)}$ are the absolute values of eddy current densities at the point $P(50,0,0)$ in the models $C$ and $D$. The conductivities $\sigma_{a}$ and $\sigma_{b}$ in Figs.6(a) and (b) are $0.7507 \times 10^{3} \mathrm{~S} / \mathrm{m}$ and $0.7507 \times 10^{7} \mathrm{~S} / \mathrm{m}$ respectively. When the skin effect is negligibly small $(\delta / W=1.5), \phi(2)$ is nearly equal to $\phi(3)$. $\mathrm{Je}_{\mathrm{e}}(2)$ is larger than $\mathrm{Je}^{(3)}$, because the eddy current path in the 2-D model $\mathrm{C}$ is shorter than that in the 3-D model $\mathrm{D}$ as shown in Fig.7. When the skin effect is significant $(\delta / W=0.015), \phi(2)$ is considerably less than $\phi(3)$, and $\mathrm{Je}^{(2)}$ is nearly equal to $\mathrm{Je}^{(3)}$, as shown in Fig.6(b). Fig.8 shows the effects of $\mathrm{L}_{1}$ on the ratios
$\phi(2) / \phi(3)$ the constant voltage excitation. When the skin effect is significant $(\delta / W=0.015), \phi(2)$ and $\mathrm{Je}^{(2)}$ are always larger than $\phi(3)$ and $\mathrm{Je}_{\mathrm{e}}(3)$, as shown in Fig.8(b). In this case, the amount of the leakage flux increases due to the opposing field of the eddy current.

The effects of the end coil on the flux in the pole piece have also been investigated. It is found that the effect of the end coil is negligible, especially

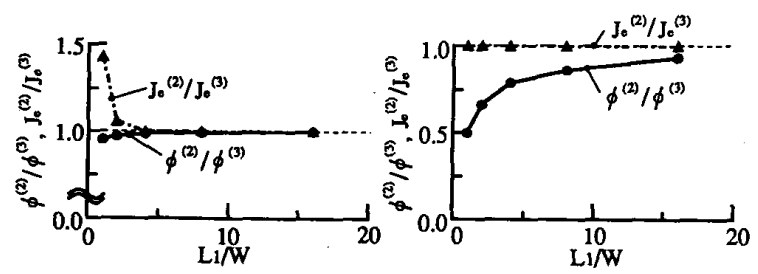

$\begin{array}{lll}\text { (a) } \delta / W=1.5 & \text { ( } \delta: \text { skin depth) } & \text { (b) } \delta / W=0.015\end{array}$

Fig.6 Effect of eddy current path on flux and eddy current density (constant current excitation, $60 \mathrm{~Hz}, \mathrm{G}=0 \mathrm{~mm}$ ).

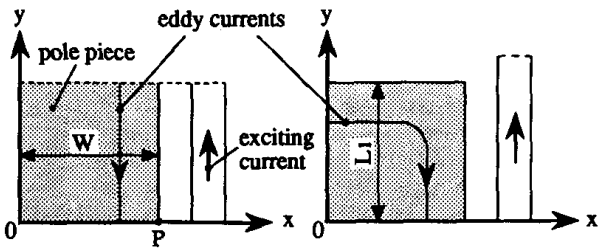

(a) model C (2-D)

(b) model D (3-D)

Fig.7 Eddy current paths.

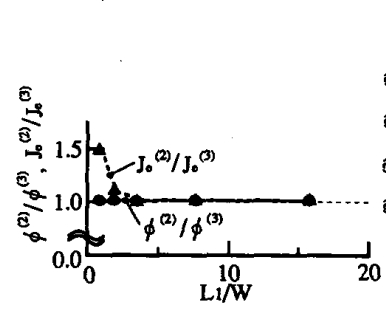

(a) $\delta / \mathrm{W}=1.5$

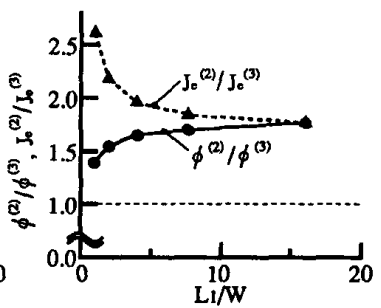

$(\delta:$ skin depth )
Fig.8 Effect of eddy current path on flux and eddy current density (constant voltage excitation, $60 \mathrm{~Hz}, \mathrm{G}=0 \mathrm{~mm}$ ). 
under the constant current excitation.

It may be concluded that if the gap length $G$ is seall and the excitation is nearly dc(skin effect is not significant), electric machines can be analyzed accurately using a 2-D model.

\section{EXPERIMENTS}

The effects of the width $L_{1}$ of the pole piece and the gap length $G$ on the flux in the core have been exained experimentally. Fig. 9 shows the experimental model. This nodel has two gaps. The solid core is made of steel. The coil has 750 ampere turns. The average flux density in the core is measured using the search coil S.

Figs. 10 and 11 show the effect of the width $L_{1}$ on the flux $\phi / 2 \mathrm{~L}_{1}$ per unit width in the core under constant current excitation(dc and $60 \mathrm{~Hz}$ ). The flux $\phi / 2 L_{1}$ in the thick core(nearly 2-D) is less than that in the thin core(3-D). It is similar to the case shown in Fig. 6 .

If the step by step method[6] is used for the analysis of the 3-D nonlinear eddy current problem, extremely long CPU time is required, because a number of iterations are necessary until periodic solutions are obtained $[6]$. Therefore, only results measured are shown in Fig. 11.

The effect of the length of the end coil on the flux is also investigated. More detailed results will be reported in another paper.

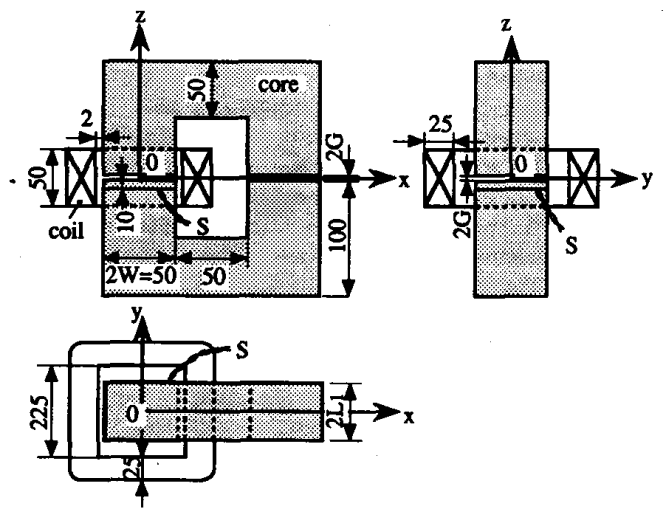

Fig.9 Experimental model.

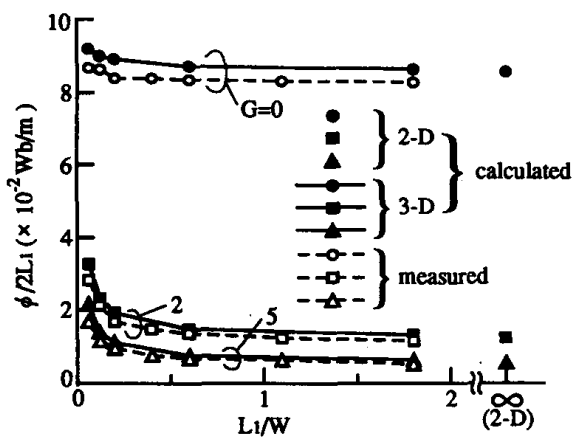

Fig. 10 Effect of width $\mathrm{L}_{1}$ on flux $\phi / 2 \mathrm{~L}_{1}$ (constant current excitation, dc).

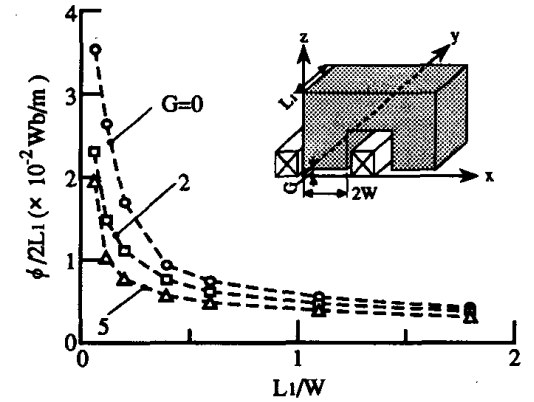

Fig.11 Effect of width $\mathrm{L}_{1}$ on flux $\phi / 2 \mathrm{~L}$ (constant current excitation, $60 \mathrm{~Hz}$, measured).

\section{v. CONCLUSIONS}

The results obtained here can be summarized as follows:

1) When the gap length is relatively large, the difference between the fluxes calculated by $2-D$ and $3-D$ analyses is significant due to the fringing flux. The flux in the pole piece obtained by 3-D analysis is larger than that by 2-D analysis under constant current excitation, and the flux obtained by 3-D analysis is less than that by $2-D$ analysis under constant voltage excitation.

2) When the eddy current flows in the thin core, a large error occurs in 2-D analysis, because there is a great difference in eddy current paths between 2-D and 3-D models.

3) The effect of the end coil is negligible, especially under the constant current excitation.

4) If the gap length $G$ is small and the excitation is nearly dc(skin depth $\delta$ is large), the 3-D model with thin core yields the same results as the 2-D model.

More systematic analysis for the effects of the permeability and the conductivity of the pole piece, and the frequency of the power source on the difference of the flux and eddy current distributions between 2-D and 3-D analyses will be reported in future work. The effects of the nonlinearity of the pole piece should also be examined.

\section{REFERENCES}

[1] T.Nakata, N.Takahashi, K.Fujiwara \& A.Ahagon: "Periodic boundary condition for 3-D magnetic field analysis and its applications to electrical machines", IEEE Trans. Magnetics, MAG-24, 6, 2694 (1988).

[2] T.Nakata and N.Takahashi: "Direct finite element analysis of flux and current distributions under specified conditions", ibid., MAG-18, 2, 325 (1982).

[3] T.Nakata, N.Takahashi, K.Fujiwara and A.Ahagon: "3-D finite element method for analyzing magnetic fields in electrical machines excited from voltage sources", ibid., MAG-24, 6, 2582 (1988).

[4] N.Takahashi, T.Nakata and K.Fujiwara: "Improvements of 3D finite element method for eddy current analysis and its application to fusion technology", Fusion Engineering and Design 9,113 (1989).

[5] T.Nakata, N.Takahashi, K.Fujiwara and T.Shiraki: "Comparison of different finite elements for 3-D eddy current analysis", ibid., MAG-26, 2, 434 (1990).

[6] T.Nakata, N.Takahashi, K. Fujiwara and A.Ahagon: "3-D Non-linear eddy current analysis using the timeperiodic finite element method", ibid., MAG-26, 5, 4150 (1989). 